CU-TP-551

\title{
Exchange Operator Formalism for Integrable Systems of Particles
}

\author{
Alexios P. Polychronakos \\ Pupin Physics Laboratories, Columbia University, \\ New York, NY 10027
}

\begin{abstract}
We formulate one dimensional many body integrable systems in terms of a new set of phase space variables involving exchange operators. The hamiltonian in these variables assumes a decoupled form. This greatly simplifies the derivation of the conserved charges and the proof of their commutativity at the quantum level.
\end{abstract}


In one spatial dimension a class of integrable many-body systems is known, referred to as the Calogero-Sutherland-Moser systems ${ }^{1-3}$. They constitute of many identical nonrelativistic particles interacting through two-body potentials of the inverse square type and its generalizations, namely the inverse sine square and the Weierstrass two-body potentials. These models are related to root systems of $A_{n}$ algebras ${ }^{4}$. Corresponding systems related to root systems of other algebras exist, but their two-body potentials are not translationally and/or permutation invariant ${ }^{5}$. We will restrict ourselves to the $A_{n}$ systems. For a comprehensive review of these systems see ref. 5 .

Many of the above systems admit a matrix formulation ${ }^{5,6}$. Using this formulation, a generalization of these systems was found recently where the particles also feel external potentials of particular types ${ }^{7}$. These systems, apart from their purely mathematical interest, are also of significant physical interest, since they are relevant to fractional statistics and anyons ${ }^{8}$, spin chain models ${ }^{9}$, soliton wave propagation ${ }^{10}$ and, indirectly, to nonperturbative two-dimensional quantum gravity ${ }^{11}$.

The purpose of this paper is to present an "exchange operator" formalism for these systems which renders their integrable structure explicit. Specifically, we will write generalized momentum operators in terms of which the integrals of motion assume a "decoupled" form. This will allow for an easy proof of commutativity at the quantum level.

Let $\left\{x_{i}, p_{i}\right\}, i=1, \ldots N$ be the coordinates and momenta of $N$ one-dimensional quantum mechanical particles, obeying canonical commutation relations, and let $M_{i j}$ be the particle permutation operators, obeying

$$
\begin{gathered}
M_{i j}=M_{j i}=M_{i j}^{\dagger}, \quad M_{i j}^{2}=1 \\
M_{i j} A_{j}=A_{i} M_{i j}, \quad M_{i j} A_{k}=A_{k} M_{i j}, \quad \text { for } k \neq i, j
\end{gathered}
$$

where $A_{i}$ is any operator (including $M_{i j}$ themselves) carrying one or more particle indices. Then define the "coupled" momentum operators

$$
\pi_{i}=p_{i}+i \sum_{j \neq i} V_{i j} M_{i j}, \quad V_{i j} \equiv V\left(x_{i}-x_{j}\right)
$$

with $V(x)$ an as yet undetermined function. Note that the $\pi_{i}$ are "good" one-particle operators, that is they satisfy (2), since the remaining particle indices in (3) appear in a permutation symmetric way. If we impose the hermiticity condition on $\pi_{i}$

$$
\pi_{i}=\pi_{i}^{\dagger}
$$


then $V(x)$ must obey

$$
V(x)^{\dagger}=-V(-x)
$$

Consider now a hamiltonian for the system which takes a free form in terms of $\pi_{i}$ 's, that is,

$$
H=\frac{1}{2} \sum_{i} \pi_{i}^{2}
$$

In terms of the original phase space variables, $H$ takes the form

$$
H=\frac{1}{2} \sum_{i} p_{i}^{2}+\frac{1}{2} \sum_{i \neq j}\left[i V_{i j}\left(p_{i}+p_{j}\right) M_{i j}+V_{i j}^{\prime} M_{i j}+V_{i j}^{2}\right]-\frac{1}{6} \sum_{i \neq j \neq k \neq i} V_{i j k} M_{i j k}
$$

In the above, $V^{\prime}(x)$ is the derivative of $V(x)$ and we defined

$$
V_{i j k}=V_{i j} V_{j k}+V_{j k} V_{k i}+V_{k i} V_{i j}
$$

$M_{i j k}$ is the generator of cyclic permutations in three indices, that is,

$$
M_{i j k}=M_{j k i}=M_{k i j}=M_{j i k}^{\dagger}=M_{i j} M_{j k}
$$

If we demand that the above expression for $H$ become the sum of an ordinary kinetic term and potential terms, the terms linear in $p_{i}$ should drop, and this will happen if

$$
V(-x)=-V(x)
$$

Finally, if we want the above hamiltonian to contain only two-body potentials, the function $V(x)$ should satisfy

$$
V(x) V(y)+V(y) V(z)+V(z) V(x)=W(x)+W(y)+W(z), \text { for } x+y+z=0
$$

where $W(x)$ is a new symmetric function. $H$ takes then the form

$$
H=\frac{1}{2} \sum_{i} p_{i}^{2}+\sum_{i<j}\left[V_{i j}^{2}+V_{i j}^{\prime} M_{i j}-W_{i j} \sum_{k \neq i, j} M_{i j k}\right]
$$

and the commutator of $\pi$ 's is evaluated to be

$$
\left[\pi_{i}, \pi_{j}\right]=\sum_{k \neq i, j} V_{i j k}\left[M_{i j k}-M_{j i k}\right]
$$


Eq. (11) is a well-known functional equation for $V$ which also emerges as a condition for factorizability of the ground state of many-body systems ${ }^{3}$. It can be readily solved through a small- $x$ expansion and all its solutions are available ${ }^{12}$. Here we consider in sequence the solutions of most interest.

Assume first that $W(x)=0$. Then (11) is solved by

$$
V(x)=\frac{l}{x}
$$

with $l$ a real parameter, and the hamiltonian takes the form

$$
H=\frac{1}{2} \sum_{i} p_{i}^{2}+\sum_{i>j} \frac{l\left(l-M_{i j}\right)}{\left(x_{i}-x_{j}\right)^{2}}
$$

Define the totally symmetric quantities

$$
I_{n}=\sum_{i} \pi_{i}^{n}
$$

Since in this case $V_{i j k}=0$ we see from (13) that the $\pi_{i}$ commute and therefore the $I_{n}$ also commute. Moreover, since they commute with all $M_{i j}$, their projections in the bosonic or fermionic subspaces of the Hilbert space also commute. In these subspaces the $M_{i j}$ simply become \pm 1 and $\frac{1}{2} I_{2}=H$ becomes the hamiltonian of a set of particles interacting through inverse square potentials of strength $l(l \mp 1)$. Further, the higher quantities $I_{n}$ projected in these subspaces become the integrals of motion of the above hamiltonian. Therefore these integrals commute in these subspaces.

To show that these integrals commute in the full Hilbert space it suffices to notice that they are local operators, since they involve derivatives of at most $n$-th degree. To know their action on the wavefunction at any point it suffices to know the wavefunction in a small neighborhood around that point. Therefore, the fact that they commute cannot depend on global information on the wavefunction, namely its symmetry or antisymmetry. Thus, if they commute for bosonic or fermionic states they must commute unconditionally.

In the case $W(x)=$ constant, the solution for $V$ is

$$
V(x)=l \cot a x, \text { or } V(x)=l \operatorname{coth} a x
$$

depending on the sign of the constant. Choosing the positive sign, and making $a=1$ by appropriate choice of units, we have in subspaces of definite symmetry

$$
H=\frac{1}{2} \sum_{i} p_{i}^{2}+\sum_{i<j} \frac{l(l \mp 1)}{\sin ^{2}\left(x_{i}-x_{j}\right)}-l^{2} \frac{N\left(N^{2}-1\right)}{6}
$$


This is the Sutherland model of particles interacting through inverse sine square potentials. In this case $V_{i j k}=l^{2}$ and thus the $\pi_{i}$ 's do not commute. To show the existence of conserved quantities, define the new operators

$$
\tilde{\pi}_{i}=\pi_{i}+l \sum_{j \neq i} M_{i j}
$$

which are also "good" one-particle operators and obey the commutation relations

$$
\left[\tilde{\pi}_{i}, \tilde{\pi}_{j}\right]=2 l\left(\tilde{\pi}_{i} M_{i j}-M_{i j} \tilde{\pi}_{i}\right)
$$

The corresponding conserved quantities $\tilde{I}_{n}$ constructed from $\tilde{\pi}_{i}$ can be shown to commute as follows:

$$
\begin{aligned}
{\left[\tilde{\pi}_{i}^{n}, \tilde{\pi}_{j}\right] } & =\sum_{\alpha=0}^{n-1} \tilde{\pi}_{i}^{\alpha}\left[\tilde{\pi}_{i}, \tilde{\pi}_{j}\right] \tilde{\pi}_{i}^{n-\alpha-1} \\
& =2 l\left(\tilde{\pi}_{i}^{n} M_{i j}-M_{i j} \tilde{\pi}_{i}^{n}\right)=2 l\left(M_{i j} \tilde{\pi}_{j}^{n}-\tilde{\pi}_{j}^{n} M_{i j}\right)
\end{aligned}
$$

and thus

$$
\begin{aligned}
{\left[\tilde{I}_{n}, \tilde{I}_{m}\right] } & =\sum_{i, j}\left[\tilde{\pi}_{i}^{n}, \tilde{\pi}_{j}^{m}\right]=\sum_{i, j} \sum_{\alpha=0}^{m-1} \tilde{\pi}_{j}^{\alpha}\left[\tilde{\pi}_{i}^{n}, \tilde{\pi}_{j}\right] \tilde{\pi}_{j}^{m-\alpha-1} \\
& =2 l \sum_{i, j} \sum_{\alpha=0}^{m-1}\left(\tilde{\pi}_{j}^{\alpha} M_{i j} \tilde{\pi}_{j}^{m+n-\alpha-1}-\tilde{\pi}_{j}^{\alpha+n} M_{i j} \tilde{\pi}_{j}^{m-\alpha-1}\right) \\
& =2 l \sum_{i, j}\left(\sum_{\alpha=0}^{m-1}-\sum_{\alpha=n}^{m+n-1}\right) \tilde{\pi}_{j}^{\alpha} M_{i j} \tilde{\pi}_{j}^{m+n-\alpha-1}
\end{aligned}
$$

Antisymmetrizing (22) explicitly in $n$ and $m$, we get

$$
\left[\tilde{I}_{n}, \tilde{I}_{m}\right]=l \sum_{i, j}\left(\sum_{\alpha=0}^{m-1}-\sum_{\alpha=n}^{m+n-1}-\sum_{\alpha=0}^{n-1}+\sum_{\alpha=m}^{m+n-1}\right) \tilde{\pi}_{j}^{\alpha} M_{i j} \tilde{\pi}_{j}^{m+n-\alpha-1}=0
$$

Therefore the $\tilde{I}_{n}$ commute. In subspaces of definite symmetry, on the other hand, they reduce to combinations of $I_{n}$, e.g.,

$$
\tilde{I}_{1}=I_{1} \mp l N(N-1), \quad \tilde{I}_{2}=I_{2} \mp 2 l(N-1) I_{1}+l^{2} N(N-1)^{2}, \text { e.t.c. }
$$

Therefore the $I_{n}$ commute as well. By repeating the above argument, or simply by analytic continuation, we can also deal with the coth ax solution in (17) which corresponds to the inverse hyperbolic sine square potential. 
A singular solution of (11) for $W(x)$ a negative constant is

$$
V(x)=l \operatorname{sign}(x)
$$

which leads to the well-known system of particles with mutual delta-function potentials ${ }^{13}$. It can be treated as above, with some extra care to possible singularities.

Finally, consider the operators

$$
h_{i}=\left(\pi_{i}+i \omega x_{i}\right)\left(\pi_{i}-i \omega x_{i}\right) \equiv a_{i}^{\dagger} a_{i}
$$

for $V(x)$ as in (14). Using the commutativity of the $\pi_{i}$ in this case as well as

$$
\left[x_{i}, \pi_{j}\right]=i \delta_{i j}\left(1+l \sum_{k \neq i} M_{i k}\right)-i\left(1-\delta_{i j}\right) l M_{i j}
$$

we find

$$
\begin{gathered}
{\left[a_{i}, a_{j}\right]=\left[a_{i}^{\dagger}, a_{j}^{\dagger}\right]=0} \\
{\left[a_{i}, a_{j}^{\dagger}\right]=-2 l \omega M_{i j} \quad(\text { for } \mathrm{i} \neq \mathrm{j})}
\end{gathered}
$$

and thus

$$
\left[h_{i}, h_{j}\right]=-2 l \omega\left(h_{i} M_{i j}-M_{i j} h_{i}\right)
$$

We observe that the commutation relations of the $h_{i}$ are similar to the ones of $\tilde{\pi}_{i}$ in $(20)$. Therefore the quantities $I_{n}$ defined now

$$
I_{n}=\sum_{i} h_{i}^{n}
$$

can be shown to commute in a way similar to the one for the $\tilde{I}_{n}$. In particular, the hamiltonian $H=\frac{1}{2} I_{1}$ in the bosonic or fermionic subspace becomes

$$
H=\frac{1}{2} \sum_{i} p_{i}^{2}+\sum_{i>j} \frac{l(l \mp 1)}{\left(x_{i}-x_{j}\right)^{2}}+\frac{1}{2} \sum_{i} \omega^{2} x_{i}^{2}-N \frac{\omega}{2} \pm l \frac{N(N-1)}{2} \omega
$$

This is the Calogero model of harmonic plus inverse square potentials, and we have derived its integrals of motion.

Notice that the constant terms appearing in (18) and (31) are the negative of the ground state energy of the corresponding hamiltonians, thus shifting the ground state 
energy to zero. In fact we can easily find the ground state wavefunction noticing that the above $H$ 's are positive definite and thus if we can find states $\psi_{S}$ and $\psi_{C}$ satisfying

$$
\pi_{i} \psi_{S}=0, \text { or } a_{i} \psi_{C}=0
$$

these will be the ground state. Taking them further to be bosonic, they thus must satisfy

$$
\frac{\partial_{i} \psi_{S}}{\psi_{S}}=\sum_{j \neq i} l \cot \left(x_{i}-x_{j}\right)
$$

or

$$
\frac{\partial_{i} \psi_{C}}{\psi_{C}}=-\omega x_{i}+\sum_{j \neq i} \frac{l}{x_{i}-x_{j}}
$$

respectively. By integrating (33) and (34) we easily find the Sutherland and Calogero ground state wavefunctions

$$
\psi_{S}=\prod_{i<j}\left|\sin \left(x_{i}-x_{j}\right)\right|^{l}, \quad \psi_{C}=\prod_{i<j}\left|x_{i}-x_{j}\right|^{l} e^{-\frac{1}{2} \omega \sum_{i} x_{i}^{2}}
$$

In conclusion, we see that the above formalism identifies a better set of phase space "momentum" variables, which allow for an effortless and relatively straightforward derivation of the integrability of these systems. It is also remarkable that the above proofs work directly in the quantum regime (the exchange operators $M_{i j}$ have no classical counterpart), thus circumventing the operator ordering problems encountered when constructing the quantum integrals of motion starting from the classical standpoint. It is hoped that this formalism will provide an easy proof of the quantum integrability of the systems recently found in ref. 7 , or even that it will lead to as yet uncovered new integrable systems. We hope to come back to these issues in a future publication.

This research was supported in part by a United States Department of Energy grant.

\section{REFERENCES}

1 F. Calogero, J. Math. Phys. 10, 2191 and 2197 (1969) and 12, 419 (1971).

2 J. Moser, Adv. Math. 16, 1 (1975); F. Calogero, Lett. Nuovo Cim. 13, 411 (1975); F. Calogero and C. Marchioro, Lett. Nuovo Cim. 13, 383 (1975). 
3 B. Sutherland, Phys. Rev. A4, 2019 (1971) and A5, 1372 (1972); Phys. Rev. Lett. 34, 1083 (1975).

4 M.A. Olshanetsky and A.M. Perelomov, Invent. Math. 37, 93 (1976).

5 M.A. Olshanetsky and A.M. Perelomov, Phys. Rep. 71, 314 (1981) and 94, 6 (1983).

6 D. Kazdan, B. Kostant and S. Sternberg, Comm. Pure Appl. Math. 31, 481 (1978).

7 A.P. Polychronakos, Phys. Lett. B266, 29 (1991); Columbia preprints CU-TP-527, July 1991, to appear in Phys. Lett. B and CU-TP-537, October 1991, to appear in Phys. Lett. B.

8 J.M. Leinaas and J. Myrheim, Phys. Rev. B37, 9286 (1988); A.P. Polychronakos, Nucl. Phys. B324, 597 (1989) and Phys. Lett. B264, 362 (1991).

9 F.D.M. Haldane, Phys. Rev. Lett. 60, 635 (1988) and 66, 1529 (1991); B.S. Shastry, Phys. Rev. Lett. 60, 639 (1988); F. Gebhard and A.E. Ruckenstein, Phys. Rev. Lett. 68, 244 (1992).

10 H.H. Chen, Y.C. Lee and N.R. Pereira, Phys. Fluids 22(1) , 187 (1979).

11 V.A. Kazakov, Random Surfaces and Quantum Gravity, Cargese Lectures 1990, eds. O. Alvarez et. al.

12 F. Calogero, Lett. Nuovo Cim. 13, 507 (1975).

13 E.H. Lieb and W. Liniger, Phys. Rev. 130, 1605 (1963); C.N. Yang, Phys. Rev. Lett. 19, 1312 (1967) and Phys. Rev. 168, 1920 (1968). 